\title{
Are All Primary Immunodeficiency Disorders Inborn Errors of Immunity?
}

\author{
Rohan Ameratunga ${ }^{1,2,3^{*}}$, Hilary Longhurst ${ }^{1,4}$, Klaus Lehnert ${ }^{5}$, Richard Steele ${ }^{2}$, \\ Emily S. J. Edwards ${ }^{6}$ and See-Tarn Woon ${ }^{2}$
}

${ }^{1}$ Department of Clinical Immunology, Auckland Hospital, Auckland, New Zealand, ${ }^{2}$ Department of Virology and Immunology, Auckland Hospital, Auckland, New Zealand, ${ }^{3}$ Department of Molecular Medicine and Pathology, School of Medicine, Faculty of Medical and Health Sciences, University of Auckland, Auckland, New Zealand, ${ }^{4}$ Department of Medicine, School of Medicine, Faculty of Medical and Health Sciences, University of Auckland, Auckland, New Zealand, ${ }^{5}$ School of Biological Sciences, University of Auckland, Auckland, New Zealand, ${ }^{6}$ B Cell Differentiation Laboratory, Department of Immunology and Pathology, Central Clinical School, Monash University, Melbourne, VIC, Australia

\section{OPEN ACCESS}

Edited by:

Frédéric Rieux-Laucat, U1163 Institut Imagine (INSERM),

France

Reviewed by:

Ekaterini Simoes Goudouris, Federal University of Rio de Janeiro,

Brazil

${ }^{*}$ Correspondence:

Rohan Ameratunga rohana@adhb.govt.nz

Specialty section: This article was submitted to Primary Immunodeficiencies, a section of the journal

Frontiers in Immunology

Received: 08 May 2021 Accepted: 01 June 2021

Published: 21 July 2021

Citation: Ameratunga $R$, Longhurst $H$, Lehnert K, Steele R, Edwards ESJ

and Woon S-T (2021) Are

All Primary Immunodeficiency Disorders Inborn Errors of Immunity?

Front. Immunol. 12:706796. doi: 10.3389/fimmu.2021.706796
Keywords: inborn error of immunity, primary immunodeficiency, THI, common variable immunodeficiency disorders, THA

\section{INTRODUCTION}

It is almost 70 years since the first description of Bruton's agammaglobulinemia (1). In the last decade there has been a rapid increase in the rate of discovery of new genetic defects in primary immunodeficiency disorders (PIDs), largely due to the advent of Next Generation Sequencing (NGS) $(2,3)$. NGS utilises massively parallel sequencing to analyse either the exome (WES) or the entire genome (WGS). The International Union of Immunological Societies (IUIS) expert committee of the WHO has curated over 400 such disorders (2).

This sequencing revolution has had profound benefits (and some disadvantages) for patients and their clinicians as well as scientists seeking to identify new disorders $(4,5)$. As termed by Robert Good, these "experiments of nature", have offered unique scientific insights into functioning of the immune system (6).

The many overlapping benefits of genetic confirmation for patients include certainty of diagnosis, prognostic insights and specific treatments (5). It has ushered in the era of personalised medicine. Identification of a gain for function mutation of PIK3CD for example, may lead to specific treatments such as idelalisib in addition to subcutaneous or intravenous immunoglobulin (SCIG/IVIG) replacement.

With the rapid increase in the discovery of new genetic defects, there has been a move to name these conditions inborn errors of immunity (IEI) (2). The Merriam Webster dictionary states Inborn is "being a part of the innermost nature of a person or thing" and synonyms include congenital, hereditary and inherited. Inborn thus implies these conditions are genetic and inherited, which will be transmitted to future generations. Errors in this context infer mutations, which are pathogenic and underlie the phenotype of the patient.

Although there is an argument for changing the name from PIDs to IEI, or using these terms interchangeably, there are several caveats. Three of the most common conditions, which numerically comprise the majority of patients with PIDs, do not currently have a definable genetic basis; IgA deficiency (IgAD), Common Variable Immunodeficiency Disorders (CVID) and Transient 
Hypogammaglobulinemia of Infancy (THI). Other wellrecognised conditions, which do not have a genetic explanation at this time, include Good's syndrome and CD4 lymphopenia. Even within well-defined phenotypes such as agammaglobulinemia with absent B cells or Severe Combined Immunodeficiency (SCID), not all patients have a genetic explanation for their disorder (Figure 1). This is a perspective on why these terms are not currently interchangeable and why it may be premature to abandon the term PID in favour of IEI (Table 1).

\section{IGA DEFICIENCY}

IgA deficiency (IgAD) is the most common PID in humans. IgAD can occur in the context of other well-defined PIDs such as SCID or X-linked hyper IgM syndrome or as a sporadic disorder. The frequency of sporadic IgAD may be as high as 1:358 in blood donors (7). The majority of patients with sporadic IgAD are asymptomatic, presumably because other arms of the immune system can compensate for the lack of IgA in serum and on mucosal surfaces. The genetic basis for sporadic IgAD is not understood (13). While there is an increased proportion of IgAD patients with TNFRSF13B/ TACI mutations, the prevalence of such variants exceed that of sporadic IgAD (14). Application of the American College of
Medical Genetics (ACMG) criteria indicates TACI mutations are not the cause of sporadic IgAD (15).

\section{COMMON VARIABLE IMMUNODEFICIENCY DISORDERS}

CVIDs are the most common symptomatic PID in adults and children. The prevalence of CVID varies between 1:25 000 to less than 1:100 000 in the general population. Although there may be ascertainment bias, these disorders are less common in Asian and African populations. Reports from developed Asian countries with advanced healthcare systems, such as Taiwan, Japan or South Korea indicate a very low frequency of CVID (16-19). In contrast, a recent study has shown an unexpected high prevalence of CVID in the indigenous Māori of New Zealand (20). The basis for these ethnic-specific rates is not known.

By definition, the genetic causes for CVID are unknown (21-23). There are now over forty genetic variants which are associated with CVID $(3,8,24)$. Some of these are causative (NFKB1, $N F K B 2$ etc) while others predispose to or modify disease severity (TNFRSF13B/TACI, TNFRSF13C/BAFFR, MSH5 etc.) in CVID. Sometimes mutations in genes such as $R A G$ can lead to atypical presentations which might be considered CVID-mimics. If patients have a causative genetic defect, they are now deemed

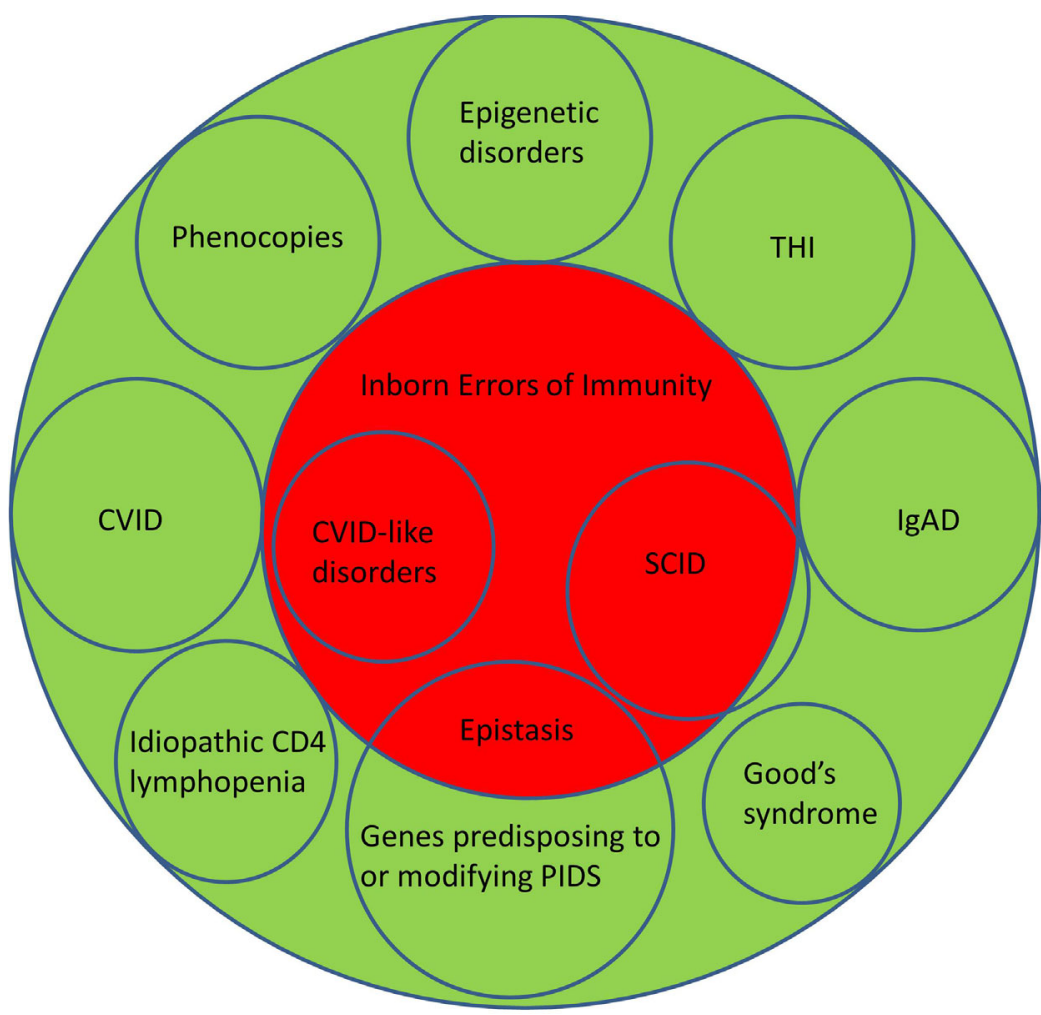

FIGURE 1 | Illustrating the relationship between Primary Immunodeficiency Disorders (green) and Inborn Errors of Immunity (red). CVID, Common Variable Immunodeficiency Disorders; IgAD, IgA deficiency; SCID, Severe Combined Immunodeficiency; THI, Transient Hypogammaglobulinemia of Infancy. 
TABLE 1 | PIDs for which a causative genetic basis has not been identified in all patients.

\begin{tabular}{|c|c|c|}
\hline Disorder & Genetic basis & Comment \\
\hline IgA deficiency & Unknown & Commonest PID (7) \\
\hline CVID & $\begin{array}{l}\text { Unknown } \\
\text { (by definition) }\end{array}$ & Patients with causative genetic defects are reclassified as CVID-like disorders $(3,8)$ \\
\hline $\mathrm{THI}$ & Unknown & Retrospective diagnosis (9) \\
\hline Idiopathic CD4 lymphopenia & Unknown & Increasingly recognised with the advent of new-born screening (10) \\
\hline Good's syndrome & Unknown & Thymic abnormalities may contribute to the combined immunodeficiency (11) \\
\hline SCID (and many other similarly well- characterised disorders) & Mostly known & Not all patients with a SCID phenotype have an identifiable mutation \\
\hline Phenocopies & Acquired disorders & Can be identified by genetic sequencing (2) \\
\hline Epigenetic defects & Acquired disorders & Difficult to identify (12) \\
\hline
\end{tabular}

CVID, Common Variable Immunodeficiency Disorders; SCID, Severe Combined Immunodeficiency; THI, Transient Hypogammaglobulinemia of Infancy.

to have a CVID-like disorder with their own specific mutation. Patients with CVID-like disorders could be considered to have an IEI (Figure 1).

In consanguineous societies, the proportion of patients with CVID-like disorders is approximately $60-70 \%(25,26)$. Patients from such countries have highly penetrant autosomal recessive monogenic disorders often presenting in childhood. In contrast, only $25 \%$ patients from non-consanguineous populations have an underlying causative mutation, mostly late-onset autosomal dominant disorders (27). In non-consanguineous societies, this leaves $75 \%$ of CVID patients without a genetic explanation and IEI would seem an incorrect term for these patients.

CVID-like disorders are characterised by marked genetic, allelic and phenotypic heterogeneity (8). It is apparent that several causative genetic defects lead to the same phenotype of recurrent infections and autoimmune disorders (locus heterogeneity, genocopy). Similarly, there can be marked phenotypic variation in the same family carrying the identical mutation. In one of the three families where mutations of NFKB1 were first described, one brother was completely asymptomatic, while his sister passed away from late onset combined immunodeficiency (LOCID). Other members of the family had predominantly autoimmune disorders $(28,29)$. This phenotypic heterogeneity of CVID-like disorders is compatible with IEI as the majority of patients with causative genetic defects would be expected to become symptomatic at some stage of their lives. It is likely environmental triggers such as Herpes simplex infections may alter their prognostic trajectory.

To complicate the genetics of CVID and CVID-like disorders, a group of genes predispose to or modify disease severity in CVID and CVID-like disorders (30). These genes include TNFRSF13B/TACI, TNFRSF13C/BAFFR, MSH5 etc. While there is a higher prevalence of mutations of these genes in patients with CVID and CVID-like disorders, their frequency in the general population far exceeds that of CVID (31). The majority of healthy individuals carrying these mutations will not suffer CVID. While these could be considered variants and are inherited, they are not causative and should not be considered IEI. The ACMG criteria cannot be applied to genes which predispose to or modify CVID (15).

This was recently illustrated in a family presenting with a severe CVID-like disorder (32). The proband had mutations of both TNFRSF13B/TACI as well as TCF3. Family studies showed it was the TCF3 mutation, which segregated with the CVID-like disorder, while the TNFRSF13B/TACI mutation modified the disorder in an epistatic fashion (33). Epistasis is the synergistic interaction of two or more genes which can modify disease severity or lead an entirely different phenotype (34). This family illustrates the complexity of CVID and CVID-like disorders and the need for accurate description of the underlying genetics. Individuals from this kindred carrying the TCF3 mutation could be considered to have an IEI but not those with TNFRSF13B/ TACI, which modified the severity of the disorder.

\section{TRANSIENT HYPOGAMMAGLOBULINEMIA OF INFANCY}

Transient hypogammaglobulinemia of infancy is an important cause of reduced immunoglobulin levels in early life (35). Current thinking is that there is delayed maturation of IgG production in these infants. By definition, the IgG normalises over time and THI is a retrospective diagnosis. Recently it has been shown that the majority of patients do not normalise their IgG until after four years of age (9).

The genetic basis of THI is currently not understood. This is likely to be a common PID but may not be recognised as many patients are asymptomatic. Again, it is inaccurate to term these infants as having an IEI.

\section{OTHER IDIOPATHIC DISORDERS WITH A PROBABLE GENETIC BASIS}

There are many other well-defined disorders where the genetic basis has not been identified. This includes patients with Good's syndrome, a combination of thymoma, CD4 lymphopenia and hypogammaglobulinemia (11). Most patients with Good's syndrome are diagnosed following a CT scan of the thorax, when the thymoma is identified.

Following the advent of new-born screening it has become apparent many patients with low numbers of $\mathrm{T}$ cell receptor excision circles (TRECs) have idiopathic $\mathrm{T}$ cell lymphopenia 
rather than Severe Combined Immunodeficiency (SCID) (10). The long-term prognosis of these infants is not known. The genetic basis for persistent idiopathic $\mathrm{T}$ cell lymphopenia is not understood and it would not be accurate to label this condition as an IEI (36).

\section{PHENOCOPIES}

The WHO/IUIS committee has recognised a group of disorders called phenocopies, which do not easily fit with IEI (2). Phenocopies are caused by discrete clones of cells, which proliferate and reproduce the phenotype of the disorder. There are many examples of these somatic disorders including the autoimmune lymphoproliferative syndrome (ALPs). While these conditions have a genetic basis, they cannot be inherited and do not meet the inborn component of IEI. It may still be appropriate to call them PIDs as there is no secondary cause.

\section{EPIGENETIC DISORDERS}

Monozygotic twins discordant for CVID have been described (12). The authors speculated the explanation was epigenetic changes in several genes including TCF3. It will be important to show these methylation patterns are stable over time and segregate with the phenotype of the affected twin. It seems likely other PIDs will be identified in the future, where epigenetic changes are the basis of the disorder. Epigenetic changes are not considered IEIs but would be considered PIDs.

\section{DISCUSSION}

This essay has examined the interchangeability of the terms PID and IEI. There are many reasons why the term primary immunodeficiency disorder is currently preferred to inborn error of immunity. This is not merely a matter of semantics, as it has important implications for scientific accuracy as well as clinical management of these patients.

Unlike IEI, PID does not necessarily imply there is an inherited genetic basis for the disorder and could include phenocopies. The aberrant clone of cells harbouring the mutation is directly responsible for the clinical manifestations of disease. In secondary causes such as lymphoma, the immune defect is consequent to the malignant process. Phenocopies can thus be considered PIDs as they are the cause of the disorder, but not IEIs as they cannot be inherited despite having a genetic basis.

Epigenetic disorders cannot be easily identified by current sequencing technologies but may be more prevalent than is currently perceived. If more disorders are shown to have an epigenetic basis, the term IEI will be inappropriate as these conditions cannot be inherited. They will however remain PIDs as there is no secondary cause for the disorder.
Scientific integrity is important. Given that the genetic basis of CVID is by definition not known, stating patients with CVID have an IEI is inaccurate. In addition to scientific inaccuracy, semantics can also adversely affect patient care. As seen in the description of CVID and CVID-like disorders, the genetics of PIDs are complex $(3,24,37)$. It is very important for patients to be appropriately counselled, particularly in non-consanguineous populations that there is a greater chance a causative mutation will not be identified. Such genetic counselling is an essential part of pre-analytical testing. If patients with CVID are advised they have an IEI, it may create unrealistic expectations that a causative genetic defect will be identified.

Furthermore, it may create anxiety as IEI more than PID might indicate the disorder will be passed to the next generation. PIDs, like many familial conditions are associated with parental guilt. This may be exacerbated if the genetic basis is not identified after testing. PIDs in contrast do not imply all patients have a genetic basis for their disorder, as there are other pathogenic mechanisms. Classifying CVID as a subset of PIDs is both scientifically accurate and is more helpful in managing patient expectations during pre-analytical counselling (Figure 1).

IEI also implies greater scientific understanding than is currently the case for many disorders. Asymptomatic IgAD likely comprises the majority of patients with PIDs. As noted above, the genetic basis for sporadic IgAD is unknown. As with CVID, it is scientifically more accurate to term these conditions PIDs than IEIs. Numerically, symptomatic and asymptomatic patients with sporadic IgAD far exceed all other PIDs and cannot be termed IEIs.

Similarly, although rare familial cases of THI have been described in siblings, the vast majority are sporadic (9). The genetic basis for familial THI is unknown. The use of the term IEI could cause unnecessary parental anxiety and guilt if the index child has suffered invasive infections and has needed SCIG/IVIG. In this context IEI implies a greater understanding of THI than is the case.

One argument for using IEI is the case of gain of function (GOF) mutations leading to autosomal dominant disorders. Such heterozygous cases lead to a phenotype, which is often very different from patients with loss of function mutations in the same gene. Although at first glance, the term PID may not seem to fit well, these conditions could be considered to be an immunodeficiency of regulatory elements of gene function. A similar argument could be made for patients with CVID-like disorders presenting with autoimmunity with a minimal history of infections.

Inspite of these differences, there are however areas of agreement between the terms PID and IEI. Both exclude secondary causes such as infections, gut disease, malignancy, renal immunoglobulin loss etc. (38). Generally secondary causes such as malignancy can be easily distinguished from PIDs. The age of the patient is helpful in considering the likely secondary causes. The passage of time and family history can usually help distinguish secondary causes from a primary disorder.

In the last decade, there has been rapid progress in the identification of the genetic basis of many PIDs. If there is 
another future genetic revolution akin to NGS, it is possible all patients with PIDs will have a genetic diagnosis. Apart from patients with phenocopies and epigenetic changes, the two terms PID and IEI may be interchangeable.

All current definitions of CVID exclude a known cause for hypogammaglobulinemia (21-23). Counterintuitively, if the genetic basis of CVID was understood in all patients, the disorder will cease to exist (8). All such patients with CVID-like disorders will have their own genetic defect/IEI. It would seem reasonable to revisit this topic periodically, particularly if there have been major advances in technology.

\section{REFERENCES}

1. Bruton OC. Agammaglobulinemia (Congenital Absence of Gamma Globulin); Report of a Case. In: The Medical Annals of the District of Columbia, vol. 22. (1953). p. 648-50.

2. Tangye SG, Al-Herz W, Bousfiha A, Chatila T, Cunningham-Rundles C, Etzioni A, et al. Human Inborn Errors of Immunity: 2019 Update on the Classification From the International Union of Immunological Societies Expert Committee. J Clin Immunol (2020) 40(1):24-64. doi: 10.1007/ s10875-019-00737-x

3. Edwards ESJ, Bosco JJ, Ojaimi S, O’Hehir RE, van Zelm MC. Beyond Monogenetic Rare Variants: Tackling the Low Rate of Genetic Diagnoses in Predominantly Antibody Deficiency. Cell Mol Immunol (2021) 18:588-603.

4. Ameratunga R, Woon ST, Neas K, Love DR. The Clinical Utility of Molecular Diagnostic Testing for Primary Immune Deficiency Disorders: A Case Based Review. Allergy Asthma Clin Immunol: Off J Can Soc Allergy Clin Immunol (2010) 6(1):12. doi: 10.1186/1710-1492-6-12

5. Ameratunga R, Woon ST, Brewerton M, Koopmans W, Jordan A, Brothers S, et al. Primary Immune Deficiency Disorders in the South Pacific: The Clinical Utility of a Customized Genetic Testing Program in New Zealand. Ann NY Acad Sci (2011) 1238:53-64. doi: 10.1111/j.1749-6632.2011.06238.x

6. Good RA. Experiments of Nature in the Development of Modern Immunology. Immunol Today (1991) 12(8):283-6. doi: 10.1016/0167-5699 (91)90127-F

7. Clark JA, Callicoat PA, Brenner NA, Bradley CA, Smith DM Jr. Selective IgA Deficiency in Blood Donors. Am J Clin Pathol (1983) 80(2):210-3.

8. Ameratunga R, Allan C, Woon ST. Defining Common Variable Immunodeficiency Disorders in 2020. Immunol Allergy Clin North Am (2020) 40(3):403-20. doi: 10.1016/j.iac.2020.1003.1001

9. Ameratunga R, Ahn Y, Steele R, Woon ST. Transient Hypogammaglobulinemia of Infancy: Many Patients Recover in Adolescence and Adulthood. Clin Exp Immunol (2019) 198(2):224-32. doi: 10.1111/cei.13345

10. Albin-Leeds S, Ochoa J, Mehta H, et al. Idiopathic T Cell Lymphopenia Identified in New York State Newborn Screening. Clin Immunol (2017) 183:36-40. doi: 10.1016/j.clim.2017.07.002

11. Malphettes M, Gérard L, Galicier L, Boutboul D, Asli B, Szalat R, et al. Good Syndrome: An Adult-Onset Immunodeficiency Remarkable for Its High Incidence of Invasive Infections and Autoimmune Complications. Clin Infect Dis (2015) 61(2):e13-9. doi: 10.1093/cid/civ269

12. Rodriguez-Cortez VC, Del Pino-Molina L, Rodriguez-Ubreva J, Ciudad L, Gomez-Cabrero D, Company C, et al. Monozygotic Twins Discordant for Common Variable Immunodeficiency Reveal Impaired DNA Demethylation During Naive-to-Memory B-Cell Transition. Nat Commun (2015) 6:7335. doi: $10.1038 /$ ncomms 8335

13. Swain S, Selmi C, Gershwin ME, Teuber SS. The Clinical Implications of Selective IgA Deficiency. J Trans Autoimmun (2019) 2:100025. doi: 10.1016/ j.jtauto.2019.100025

14. Castigli E, Wilson SA, Garibyan L, Garibyan L, Rachid R, Bonilla F, et al. TACI Is Mutant in Common Variable Immunodeficiency and IgA Deficiency. Nat Genet (2005) 37(8):829-34. doi: 10.1038/ng1601

15. Ameratunga R, Allan C, Lehnert K, Woon ST. Perspective: Application of the American College of Medical Genetics Variant Interpretation Criteria to

\section{AUTHOR CONTRIBUTIONS}

RA wrote the first draft. All authors contributed to the article and approved the submitted version.

\section{ACKNOWLEDGMENTS}

We thank our patients for participating in studies for the benefit of others. We hope these concepts will help them in the future.

Common Variable Immunodeficiency Disorders. Clin Rev Allergy Immunol (2021). doi: 10.1007/s12016-020-08828-z

16. Tseng CW, Lai KL, Chen DY, Lin $\mathrm{CH}$, Chen HH. The Incidence and Prevalence of Common Variable Immunodeficiency Disease in Taiwan, A Population-Based Study. PloS One (2015) 10(10):e0140473. doi: 10.1371/ journal.pone. 0140473

17. Rhim JW, Kim KH, Kim DS, Kim BS, Kim JS, Kim CH, et al. Prevalence of Primary Immunodeficiency in Korea. J Korean Med Sci (2012) 27(7):788-93. doi: $10.3346 / \mathrm{kms} .2012 .27 .7 .788$

18. Ishimura $M$, Takada $H$, Doi $T$, Imai $K$, Sasahara $Y$, Kanegane $H$, et al. Nationwide Survey of Patients With Primary Immunodeficiency Diseases in Japan. J Clin Immunol (2011) 31(6):968-76. doi: 10.1007/s10875-011-9594-7

19. Son S, Kang JM, Hahn Y, Ahn K, Kim YJ. Systematic Review of Literature and Analysis of Big Data From the National Health Insurance System on Primary Immunodeficiencies in Korea. Clin Exp Pediatr (2021) 64:141-8.

20. Ameratunga R, Jordan A, Cavadino A, Ameratunga S, Hills T, Steele R, et al. Bronchiectasis Is Associated With Delayed Diagnosis and Adverse Outcomes in the New Zealand Common Variable Immunodeficiency Disorders Cohort Study. Clin Exp Immunol (2021) 204(3):352-60. doi: 10.1111/cei.13595

21. Ameratunga R, Woon ST, Gillis D, Koopmans W, Steele R. New Diagnostic Criteria for Common Variable Immune Deficiency (CVID), Which May Assist With Decisions to Treat With Intravenous or Subcutaneous Immunoglobulin. Clin Exp Immunol (2013) 174(2):203-11. doi: 10.1111/ cei. 12178

22. Bonilla FA, Barlan I, Chapel H, Costa-Carvalho BT, Cunningham-Rundles C, de la Morena MT, et al. International Consensus Document (Icon): Common Variable Immunodeficiency Disorders. J Allergy Clin Immunol Pract (2016) 4 (1):38-59. doi: 10.1016/j.jaip.2015.07.025

23. Seidel MG, Kindle G, Gathmann B, Quinti I, Buckland M, van Montfrans J, et al. The European Society for Immunodeficiencies (ESID) Registry Working Definitions for the Clinical Diagnosis of Inborn Errors of Immunity. J Allergy Clin Immunol Pract (2019) 7:1763-70.

24. Aggarwal V, Banday AZ, Jindal AK, Das J, Rawat A. Recent Advances in Elucidating the Genetics of Common Variable Immunodeficiency. Genes Dis (2020) 7:26-37. doi: 10.1016/j.gendis.2019.10.002

25. Abolhassani H, Hammarstrom L, Cunningham-Rundles C. Current Genetic Landscape in Common Variable Immune Deficiency. Blood (2020) 135:65667. doi: 10.1016/B978-0-12-816768-7.00018-1

26. Al-Herz W, Chou J, Delmonte OM, Massaad MJ, Bainter W, Castagnoli R, et al. Comprehensive Genetic Results for Primary Immunodeficiency Disorders in a Highly Consanguineous Population. Front Immunol (2018) 9:3146. doi: 10.3389/fimmu.2018.03146

27. Maffucci P, Filion CA, Boisson B, Itan Y, Shang L, Casanova JL, et al. Genetic Diagnosis Using Whole Exome Sequencing in Common Variable Immunodeficiency. Front Immunol (2016) 7:220. doi: 10.3389/fimmu.2016.00220

28. Fliegauf M L, Bryant V, Frede N, Slade C, Woon S-T, Lehnert K, et al Haploinsufficiency of the NF-kbl Subunit p50 in Common Variable Immunodeficiency. Am J Hum Genet (2015) 97(3):389-403. doi: 10.1016/ j.ajhg.2015.07.008

29. Ameratunga R, Ahn Y, Jordan A, Lehnert K, Brothers S, Woon ST. Keeping it in the Family: The Case for Considering Late Onset Combined Immunodeficiency a Subset of Common Variable Immunodeficiency 
Disorders. Expert Rev Clin Immunol (2018) 14(7):549-56. doi: 10.1080/ 1744666X.2018.1481750

30. Ameratunga R, Woon ST. Perspective: Evolving Concepts in the Diagnosis and Understanding of Common Variable Immunodeficiency Disorders (CVID). Clin Rev Allergy Immunol (2020) 59:109-21. doi: 10.1007/s12016-019-08765-6

31. Pan-Hammarstrom Q, Salzer U, Du L, Bjorkander J, Cunningham-Rundles C, Nelson DL, et al. Reexamining the Role of TACI Coding Variants in Common Variable Immunodeficiency and Selective IgA Deficiency. Nat Genet (2007) 39 (4):429-30. doi: 10.1038/ng0407-429

32. Ameratunga R, Koopmans W, Woon ST, Leung E, Lehnert K, Slade CA, et al. Epistatic Interactions Between Mutations of TACI (TNFRSF13B) and TCF3 Result in a Severe Primary Immunodeficiency Disorder and Systemic Lupus Erythematosus. Clin Transl Immunol (2017) 6(10):e159. doi: 10.1038/cti.2017.41

33. Koopmans W, Woon ST, Brooks AE, Dunbar PR, Browett P, Ameratunga R. Clinical Variability of Family Members With the C104R Mutation in Transmembrane Activator and Calcium Modulator and Cyclophilin Ligand Interactor (TACI). J Clin Immunol (2013) 33(1):68-73. doi: 10.1007/s10875012-9793-x

34. Ameratunga R, Woon ST, Bryant VL, Steele R, Slade C, Leung E, et al. Clinical Implications of Digenic Inheritiance and Epistasis in Primary Immunodeficiency Disorders. Front Immunol (2018) 8:1965. doi: 10.3389/ fimmu.2017.01965

35. Tiller TL Jr., Buckley RH. Transient Hypogammaglobulinemia of Infancy: Review of the Literature, Clinical and Immunologic Features of 11 New Cases, and Long-Term Follow-Up. J Pediatr (1978) 92(3):347-53. doi: 10.1016/ S0022-3476(78)80417-X

36. Zhang S, Hon E, Bonagura V, Jongco A. Genetic Testing Outcomes in Infants With Idiopathic T-Cell Lymphopenia. J Allergy Clin Immunol (2020) 145(2): AB214.

37. Ameratunga R, Lehnert K, Woon ST. All Patients With Common Variable Immunodeficiency Disorders (Cvid) Should Be Routinely Offered Diagnostic Genetic Testing. Front Immunol (2019) 10:2678. doi: 10.3389/fimmu. 2019.02678

38. Duraisingham SS, Buckland MS, Grigoriadou S, Longhurst HJ. Secondary Antibody Deficiency. Expert Rev Clin Immunol (2014) 10(5):583-91. doi: 10.1586/1744666X.2014.902314

Conflict of Interest: The authors declare that the research was conducted in the absence of any commercial or financial relationships that could be construed as a potential conflict of interest.

Copyright (c) 2021 Ameratunga, Longhurst, Lehnert, Steele, Edwards and Woon. This is an open-access article distributed under the terms of the Creative Commons Attribution License (CC BY). The use, distribution or reproduction in other forums is permitted, provided the original author(s) and the copyright owner(s) are credited and that the original publication in this journal is cited, in accordance with accepted academic practice. No use, distribution or reproduction is permitted which does not comply with these terms. 